\title{
KÄHLER MANIFOLDS WITH NEGATIVE HOLOMORPHIC SECTIONAL CURVATURE, KÄHLER-RICCI FLOW APPROACH
}

\author{
RYOSUKE NOMURA
}

\begin{abstract}
Recently, Wu-Yau and Tosatti-Yang established the connection between the negativity of holomorphic sectional curvatures and the positivity of canonical bundles for compact Kähler manifolds. In this short note, we give another proof of their theorems by using the Kähler-Ricci flow.
\end{abstract}

\section{INTRODUCTION}

In this note, we provide a Kähler-Ricci flow approach to the following two theorems, which represent the relationship between the negativity of the holomorphic sectional curvature and the positivity of the canonical bundle $K_{X}$ of a compact Kähler manifold $X$.

Theorem 1.1 (WY16a, Theorem 2], [TY15, Corollary 1.3]). If $X$ admits a Kähler form with strictly negative holomorphic sectional curvature, then the canonical bundle $K_{X}$ is ample. In particular, $X$ is projective.

Theorem 1.2 ([TY15, Theorem 1.1]). If $X$ admits a Kähler form with seminegative holomorphic sectional curvature, then the canonical bundle $K_{X}$ is nef.

The original proofs of both theorems are based on the following idea, in WY16a, constructing a Kähler form $\omega_{\varepsilon} \in 2 \pi c_{1}\left(K_{X}\right)+\varepsilon[\widehat{\omega}]$ satisfying

$$
\operatorname{Ric}\left(\omega_{\varepsilon}\right)=-\omega_{\varepsilon}+\varepsilon \widehat{\omega}
$$

and considering the limiting behavior of $\omega_{\varepsilon}$ as $\varepsilon \searrow 0$. Here, $\widehat{\omega}$ is a Kähler form whose holomorphic sectional curvature is (strictly/semi-) negative. The objective of this note is to simplify the proofs by replacing $\omega_{\varepsilon}$ by the KählerRicci flow $\omega_{t}$.

These theorems are originated from the conjecture of Yau (see [HLW16, Conjecture 1.2]). For a historical background, we refer to [HLW16, WY16a, WY16b, TY15, DT16] and the references therein.

We remark that Diverio and Trapani [DT16] showed that the ampleness of $K_{X}$ can be obtained under the assumption that the holomorphic sectional curvature is semi-negative everywhere and strictly negative at one point. For the moment, we can only prove the above two theorems.

Acknowledgment. The author would like to thank his supervisor Prof. Shigeharu Takayama for various comments. This work is supported by the Program for Leading Graduate Schools, MEXT, Japan.

Key words and phrases. holomorphic sectional curvature, Kähler-Ricci flow.

Classification AMS 2010: 53C55, 32W20. 


\section{Properties of the Kähler-RicCi FLOW}

In this section, we summarize the basic properties of the (normalized) KählerRicci flow which will be used later. For more detailed exposition, we refer to the book BEG13. In the following argument, we will denote by $X$ a compact Kähler manifold of dimension $n$.

Definition 2.1. A smooth family of Kähler forms $\left\{\omega_{t}\right\}_{t \geq 0}$ is called the normalized Kähler-Ricci flow starting from $\omega_{0}$ if it satisfies the following equation:

$$
\begin{cases}\frac{\partial}{\partial t} \omega_{t} & =-\operatorname{Ric}\left(\omega_{t}\right)-\omega_{t} \\ \left.\omega_{t}\right|_{t=0} & =\omega_{0}\end{cases}
$$

By considering the cohomology class in $H^{1,1}(X, \mathbb{R})$ of $(2.2)$, the normalized Kähler-Ricci flow $\omega_{t}$ belongs to the cohomology class $\alpha_{t} \in H^{1,1}(X, \mathbb{R})$ defined by

$$
\alpha_{t}:=e^{-t}\left[\omega_{0}\right]+\left(1-e^{-t}\right) 2 \pi c_{1}\left(K_{X}\right) .
$$

The maximal existence theorem for the Kähler-Ricci flow is stated as follows.

Theorem 2.4 ([TZ06, Zha06, see also [BEG13, 3.3.1]). For any Kähler form $\omega_{0}$, the normalized Kähler-Ricci flow $\omega_{t}$ starting from $\omega_{0}$ exists uniquely for $t \in[0, T)$ and cannot extend beyond $T$. Here, $T$ is defined by

$$
T:=\sup \left\{t>0 \mid \alpha_{t} \text { defined by (2.3) is a Kähler class }\right\} \text {, }
$$

and called the maximal existence time. In particular, $\omega_{t}$ exists for $t \in[0, \infty)$ if and only if $K_{X}$ is nef, i.e. $2 \pi c_{1}\left(K_{X}\right)$ belongs to the closure of the Kähler cone of $X$.

We need the parabolic Schwarz lemma obtained by Song-Tian [ST07] applied to the identity map (see also [BEG13, 3.2.6]). This is a parabolic analogue of the Schwarz lemma due to Yau [Yau78].

Proposition 2.6. Let $\omega_{t}$ be the normalized Kähler-Ricci flow and $\widehat{\omega}$ be an arbitrary Kähler form. Then we have the following inequality:

$$
\left(\frac{\partial}{\partial t}-\Delta_{\omega_{t}}\right) \log \operatorname{tr}_{\omega_{t}}(\widehat{\omega}) \leq 1+\frac{g^{i \bar{j}}(t) g^{k \bar{l}}(t) \widehat{R}_{i \bar{j} k \bar{l}}}{\operatorname{tr}_{\omega_{t}}(\widehat{\omega})}
$$

The next Proposition due to Royden Roy80, Lemma] (see also [WWY12, Lemma 2.1]) will relate the negativity assumption on the holomorphic sectional curvature with a uniform estimate for the normalized Kähler-Ricci flow via the parabolic Schwarz lemma.

Proposition 2.7. Let $\widehat{\omega}$ be a Kähler form on $X$, and denote by $\widehat{H}$ the holomorphic sectional curvature of $\widehat{\omega}$. Assume that there exists a non-negative constant $\kappa \geq 0$ such that for any tangent vector $\xi \in T^{1,0} X$, we have

$$
\widehat{H}(\xi) \leq-\kappa \leq 0 \text {. }
$$

Then, for any Kähler form $\omega$, we have

$$
g^{i \bar{j}} g^{k \bar{l}} \widehat{R}_{i \bar{j} k \bar{l}} \leq-\kappa \frac{n+1}{2 n}\left(\operatorname{tr}_{\omega}(\widehat{\omega})\right)^{2} \leq 0,
$$

where $\omega=\sqrt{-1} g_{i \bar{j}} d z^{i} \wedge d \overline{z^{j}}$ and $\widehat{R}_{\overline{i j} k \bar{l}}$ is the curvature tensor of $\widehat{\omega}$. 


\section{Proof of Theorems viA KähleR-RicCI FLOW}

Proof of Theorem 1.2. Let $\widehat{\omega}$ be a Kähler form whose holomorphic sectional curvature is semi-negative i.e. $\kappa=0$ in (2.8). Let $\omega_{t}$ be the normalized Kähler-Ricci flow starting from arbitrary Kähler form $\omega_{0}$ on $X$. By Theorem 2.4, the nefness of $K_{X}$ is equivalent to the long time existence of $\omega_{t}$. By definition of the maximal existence time (2.5) and Theorem 2.4, it is enough to show that if $\omega_{t}$ exists for $\left[0, T_{0}\right)$ with $T_{0}<\infty$, then $\alpha_{T_{0}}$ is a Kähler class.

By Proposition 2.6 and, Proposition 2.7 we have

$$
\left(\frac{\partial}{\partial t}-\Delta_{\omega_{t}}\right)\left(\log \operatorname{tr}_{\omega_{t}}(\widehat{\omega})-t\right) \leq \frac{g^{i \bar{j}}(t) g^{k \bar{l}}(t) \widehat{R}_{i \bar{j} k \bar{l}}}{\operatorname{tr}_{\omega_{t}}(\widehat{\omega})} \leq 0 .
$$

Applying the maximum principle, for any $t \in\left[0, T_{0}\right)$, we have

$$
\operatorname{tr}_{\omega_{t}}(\widehat{\omega}) \leq e^{t} \max _{X} \operatorname{tr}_{\omega_{0}}(\widehat{\omega}) \leq e^{T_{0}} \max _{X} \operatorname{tr}_{\omega_{0}}(\widehat{\omega})=: C .
$$

This is equivalent to the following inequality which holds for any $t \in\left[0, T_{0}\right)$ :

$$
\frac{1}{C} \widehat{\omega} \leq \omega_{t} .
$$

Therefore, for any irreducible subvariety $V \subset X$ of positive dimension, the intersection number can be estimated as follows:

$$
\int_{V}\left[\alpha_{T_{0}}\right]^{\operatorname{dim} V}=\lim _{t \nearrow T_{0}} \int_{V} \omega_{t}^{\operatorname{dim} V} \geq \frac{1}{C^{\operatorname{dim} V}} \int_{V} \widehat{\omega}^{\operatorname{dim} V}>0 .
$$

By Demailly-Păun's characterization of the Kähler cone DP04, Main Theorem $0.1]$, the limiting class $\alpha_{T_{0}}$ is Kähler.

The idea of avoiding higher order estimates by using the Demailly-Păun's theorem can be found in the proof of [Zha10, Theorem 1.1].

Remark 3.2. We can also prove that $\omega_{t}$ converges to a smooth Kähler form as $t \nearrow T_{0}$, in particular $\alpha_{T_{0}}$ is Kähler. In fact, since the volume form $\omega_{t}^{n}$ is uniformly bounded from above (see [BEG13, 3.2.3]), (3.1) gives a uniform estimate for $\omega_{t}$ :

$$
\frac{1}{C} \widehat{\omega} \leq \omega_{t} \leq C^{\prime} \widehat{\omega} .
$$

Therefore we obtain the higher order estimates (see for example [BEG13, 3.2.16]), which guarantees the convergence.

Proof of Theorem 1.1. Let $\widehat{\omega}$ be a Kähler form whose holomorphic sectional curvature is strictly negative i.e. $\kappa>0$ in (2.8). By Theorem 1.2, $K_{X}$ is nef, and therefore $\omega_{t}$ exists for $t \in[0, \infty)$.

By Proposition 2.6 and Proposition 2.7, we get

$$
\left(\frac{\partial}{\partial t}-\Delta_{\omega_{t}}\right) \log \operatorname{tr}_{\omega_{t}}(\widehat{\omega}) \leq 1+\frac{g^{i \bar{j}}(t) g^{k \bar{l}}(t) \widehat{R}_{i \bar{j} k \bar{l}}}{\operatorname{tr}_{\omega_{t}}(\widehat{\omega})} \leq 1-\kappa \frac{n+1}{2 n} \operatorname{tr}_{\omega_{t}}(\widehat{\omega}) .
$$

Applying the maximum principle, for any $t \in[0, \infty)$, we have $\operatorname{tr}_{\omega_{t}}(\widehat{\omega}) \leq C$ where

$$
C:=\max \left\{\frac{2 n}{\kappa(n+1)}, \max _{X} \operatorname{tr}_{\omega_{0}}(\widehat{\omega})\right\}>0 .
$$


This gives, for any $t \in[0, \infty)$,

$$
\frac{1}{C} \widehat{\omega} \leq \omega_{t}
$$

Since $\alpha_{t}=\left[\omega_{t}\right]$ converges to $2 \pi c_{1}\left(K_{X}\right)$ as $t \rightarrow \infty$, the same argument as in the proof of Theorem 1.2 shows the ampleness of $K_{X}$.

Remark 3.5. Tian-Zhang [TZ06], and Zhang [Zha06] showed that under the assumption on the ampleness of $K_{X}$, any normalized Kähler-Ricci flow $\omega_{t}$ converges to the Kähler-Einstein metric with negative Ricci curvature.

\section{REFERENCES}

[BEG13] S. Boucksom, P. Eyssidieux, and V. Guedj (eds.), An introduction to the KählerRicci flow, Lecture Notes in Mathematics, vol. 2086, Springer, Cham, 2013, DOI: 10.1007/978-3-319-00819-6, MR: 3202578.

[DP04] J.-P. Demailly and M. Păun, Numerical characterization of the Kähler cone of a compact Kähler manifold, Ann. of Math. (2) 159 (2004), no. 3, 1247-1274, DOI: 10.4007/annals.2004.159.1247, MR: 2113021.

[DT16] S. Diverio and S. Trapani, Quasi-negative holomorphic sectional curvature and positivity of the canonical bundle, arXiv:1606.01381 [math.DG],

[HLW16] G. Heier, S. S. Y. Lu, and B. Wong, Kähler manifolds of semi-negative holomorphic sectional curvature, J. Differential Geom. 104 (2016), no. 3, 419-441, http://projecteuclid.org/euclid.jdg/1478138548, MR: 3568627.

[Roy80] H. L. Royden, The Ahlfors-Schwarz lemma in several complex variables, Comment. Math. Helv. 55 (1980), no. 4, 547-558, DOI: 10.1007/BF02566705, MR: 604712.

[ST07] J. Song and G. Tian, The Kähler-Ricci flow on surfaces of positive Kodaira dimension, Invent. Math. 170 (2007), no. 3, 609-653, DOI: 10.1007/s00222-007-0076-8, MR: 2357504.

[TY15] V. Tosatti and X. Yang, An extension of a theorem of Wu-Yau, arXiv:1506.01145[math.DG].

[TZ06] G. Tian and Z. Zhang, On the Kähler-Ricci flow on projective manifolds of general type, Chinese Ann. Math. Ser. B 27 (2006), no. 2, 179-192, DOI: 10.1007/s11401-005-0533-x, MR: 2243679.

[WWY12] P.-M. Wong, D. Wu, and S.-T. Yau, Picard number, holomorphic sectional curvature, and ampleness, Proc. Amer. Math. Soc. 140 (2012), no. 2, 621-626, DOI: 10.1090/S0002-9939-2011-10928-6, MR: 2846331,

[WY16a] D. Wu and S.-T. Yau, Negative holomorphic curvature and positive canonical bundle, Invent. Math. 204 (2016), no. 2, 595-604, DOI: 10.1007/s00222-015-0621-9, MR: 3489705 .

[WY16b] D. Wu and S.-T. Yau, A remark on our paper "Negative Holomorphic curvature and positive canonical bundle", arXiv:1609.01377 [math.DG].

[Yau78] S.-T. Yau, A general Schwarz lemma for Kähler manifolds, Amer. J. Math. 100 (1978), no. 1, 197-203, MR: 0486659.

[Zha06] Z. Zhang, Degenerate Monge-Ampere equations over projective manifolds, ProQuest LLC, Ann Arbor, MI, 2006, MR: 2717347.

[Zha10] Z. Zhang, Scalar curvature behavior for finite-time singularity of Kähler-Ricci flow, Michigan Math. J. 59 (2010), no. 2, 419-433, DOI: 10.1307/mmj/1281531465, MR: 2677630 .

Graduate School of Mathematical Sciences, The University of Tokyo 3-8-1 Komaba, Meguro-Ku, Tokyo, 153-8914, Japan.

E-mail address: nomu@ms.u-tokyo.ac.jp 